\title{
Imaging and histology in the diagnosis of multiple papillary fibroelastomas in a patient with hypertrophic obstructive cardiomyopathy. Case report
}

\author{
Alin Alexandru Ionescu ${ }^{1}$, Bogdan Rădulescu ${ }^{1,2}$, Vlad Herlea ${ }^{3}$, Ioan Miclea ${ }^{1}$, Irinel Parepa ${ }^{4}$, \\ Bogdan A. Popescu ${ }^{1,2}$, Şerban Bubenek ${ }^{1,2}$, Carmen Ginghină ${ }^{1,2}$, Ruxandra Jurcuțț,2
}

1"Prof. Dr. C.C. Iliescu" Institute of Emergency for Cardiovascular Diseases, Bucharest, 2"Carol Davila" University of Medicine and Pharmacy, Bucharest, ${ }^{3}$ Fundeni Clinical Institute, Department of Pathology, Bucharest, ${ }^{4}$ Constanţa County Hospital, Department of Cardiology, Constanta, Romania

\begin{abstract}
Papillary fibroelastomas (PFEs) are one of the most frequent primary cardiac tumors and occur more often in patients with hypertrophic obstructive cardiomyopathy (HOCM). PFEs have been linked to an increased risk of neurological events. We report a case of a 59-year-old woman with HOCM in whom echocardiography (transthoracic and transesophageal, using 2D and $3 \mathrm{D}$ techniques) revealed multiple masses in various locations in the left cardiac chambers. Surgical excision of the cardiac tumors and aortic valve replacement was performed and the pathologic report confirmed the diagnosis of PFEs. Patient followup using ultrasonography is crucial since recurrence is a possibility. Current cardiac ultrasound techniques are essential for diagnosing and for guiding the management of these conditions.

Keywords: hypertrophic cardiomyopathy; papillary fibroelastomas; transthoracic echocardiography; transesophageal echocardiography; cardiac surgery.
\end{abstract}

\section{Introduction}

Papillary fibroelastomas (PFEs) are considered to be the most frequent primary cardiac tumors [1]. Most often they are unique masses in the left cardiac chambers with a propensity for valvular surfaces [2]. PFEs are associated with an increased risk of neurological events, either stroke or transient ischemic accident (TIA), while rarely being directly responsible for valve dysfunction [1]. There is evidence that they arise more often in patients with hypertrophic cardiomyopathy (HCM) [3]. From a

Received 20.04.2017 Accepted 11.06.2017

Med Ultrason

2017, Vol. 19, No 4, 454-456

Corresponding author: Assoc. Prof. Ruxandra Jurcuţ

"Prof. Dr. C.C. Iliescu" Institute of Emergency for Cardiovascular Diseases, Department of Cardiology; "Carol Davila" University of Medicine and Pharmacy;

258, Fundeni street, 022232 Bucharest, Romania

E-mail: rjurcut@gmail.com historical perspective, a shift in their diagnosis has occurred, moving from an incidental intraoperative or autopsy discovery to ante-mortem diagnosis. This is largely due to the continuous improvement of imaging tools, such as better resolution of transthoracic echocardiography (TTE) [4-6] and a wider availability of transesophageal echocardiography (TEE) [7], using both two- and three-dimensional techniques. The only treatment which has shown clear benefits is represented by careful surgical excision of all such tumors, with excellent results, with a low incidence of both valve replacements and recurrence $[1,8]$.

\section{Case report}

We report the case of a 59-year-old woman diagnosed with hypertrophic obstructive cardiomyopathy (HOCM) at the age of 40, with irregular follow-up visits and episodes of persistent atrial fibrillation. She complained of shortness of breath related to moderate exercise and short 


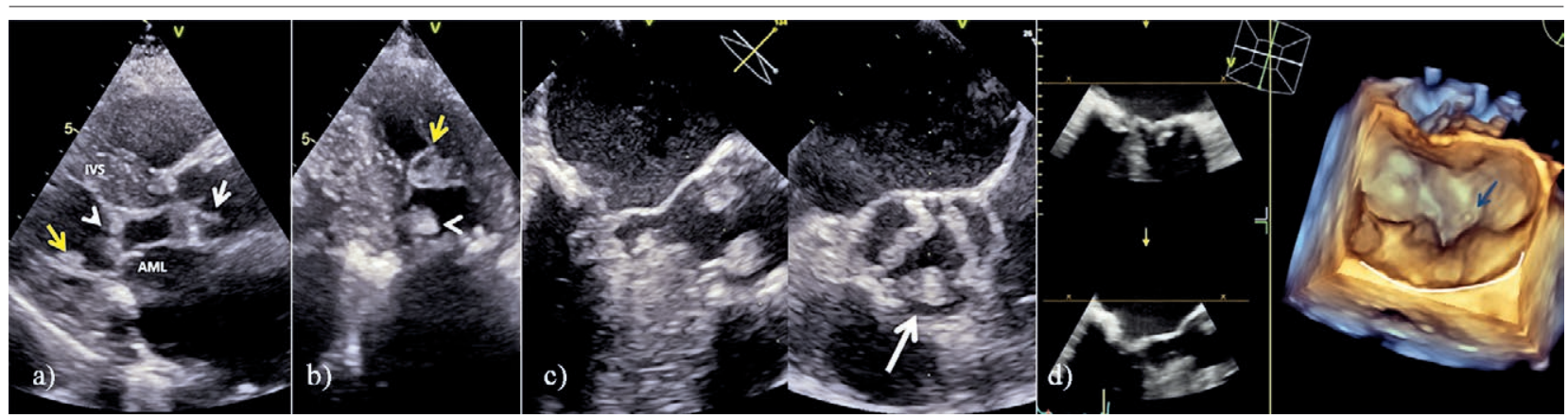

Fig 1. a) Transthoracic echocardiography, parasternal long axis view showing several mobile, round intracardiac masses, attached to the postero-medial papillary muscle (yellow arrow), in the left ventricular outflow tract at the point of contact between the interventricular septum (IVS) and the anterior mitral leaflet (AML) (white arrowhead), as well as on the aortic valve (white arrow); b) Transthoracic echocardiography, apical 4 chamber view showing one larger mass on the lateral left ventricular wall (yellow arrow) and the LVOT mass (white arrowhead); c) Transesophageal echocardiography at midesophageal level, biplane view through the aortic valve showing the presence of a large round mass on the right coronary cusp (arrow) as well as small masses on the other cusps; d) Transesophageal echocardiography at midesophageal level - three dimensional surgical view (from left atrium) - showing severe posterior wall calcification (white arch) and a small mass attached to the atrial surface of the anterior mitral leaflet (blue arrow).

periods of palpitations. Clinically, she had a 3/6 systolic heart murmur best heard at the apex, Erb's point, and over the aortic valve area. Her ECG revealed atrial fibrillation, with electric criteria for left ventricular hypertrophy. Her laboratory tests showed an increased BNP $(357 \mathrm{pg} / \mathrm{ml})$.

The TTE revealed HOCM with increased septal thickness $(16 \mathrm{~mm})$ and a left ventricle outflow tract (LVOT) dynamic pressure gradient of $34 \mathrm{mmHg}$ after Valsalva manoeuver, systolic anterior motion (SAM) of the anterior mitral leaflet (AML) for over $50 \%$ of systolic duration, left ventricular (LV) longitudinal dysfunction, moderate mitral regurgitation with a mixed mechanism (both degenerative and functional), with severe mitral annulus calcification, indirect signs of high pulmonary artery pressure, severe tricuspid regurgitation and severe biatrial enlargement. Additionally, TTE found several intracardiac masses (fig 1a,b), described as mobile, round, homogenous, with well-defined borders, of different sizes and all under $10 \mathrm{~mm}$. They were distributed throughout the structures of the left heart: near the postero-medial papillary muscle, in the LVOT at the point of contact between the interventricular septum (IVS) and the AML, and on the right coronary cusp of the aortic valve. Transesophageal echocardiography detected a supplementary small mass on the atrial side of the AML and multiple tumors were described on all aortic valve cusps (fig 1c,d). The echocardiographic appearance and distribution raised the suspicion of PFEs. Coronary computed tomography angiogram showed hypoplastic right coronary artery and no atherosclerotic lesions.

Surgical excision was recommended but the patient suffered a stroke and cardiac surgery was realized 1 month after the stroke. Aortic valve replacement and ex-

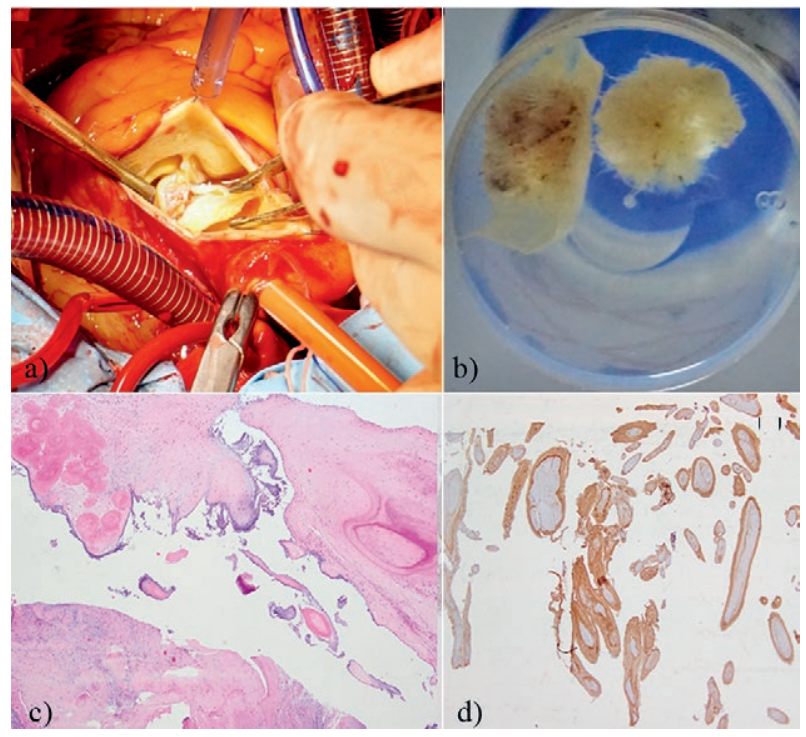

Fig 2. a) Intraoperatory exposed aortic valve with numerous tumoral masses present on all the aortic cusps; b) Sea anemone like appearance of one of the tumoral masses resected from the left ventricular outflow tract; c) Hematoxylin eosin staining (40x) showing avascular collagen core within a myxoid stroma, covered by a single layer of hyperplastic endothelial cells; d) Immunohistochemical investigation was performed and was found positive for type IV collagen.

cision of all the reported tumors were performed, including a mass near the LV apex, previously undetected by echocardiography. Histopatologic and immunhistochemical examinations (positive for CD34, S-100 protein, vimentin and type IV collagen) confirmed the diagnosis of PFEs (fig 2). Postoperative evolution at 6 months was excellent. 


\section{Discussions}

The present case illustrates the ability, and also the shortcomings, of cardiac ultrasonography to reveal intracardiac masses, to suggest the specific etiology and to guide specific treatment.

PFEs are considered to be primary cardiac tumors, more than 95\% arising in the left heart [2] and about $77 \%$ being located on the valvular surfaces (usually from the mid portion of the cusps). Their size can vary widely, from 1-2 $\mathrm{mm}$ to $70 \mathrm{~mm}$ [9], but are usually around $10 \mathrm{~mm}$ [10]. A study which included 19 patients with PFEs and HOCM [3] found that, in this patient population, PFEs are more frequent $(0.28 \%$ versus $0.02 \%$ in the overall population) and are more likely to occur on the aortic valve (47\%) and along the LVOT (42\%), as was the case of our patient. While rare and relatively small, PFEs can have devastating clinical consequences. Thromboembolic events in all arterial territories can be associated with left cardiac chambers PFEs [2]. Even though most often they are solitary lesions, in rare instances such as in the case of our patient, multiple PFEs can be present, so a thorough inspection of all cardiac chambers is mandatory.

On echocardiography, these tumors are usually small, with well-demarcated borders, with various shapes (round, oval or irregular), with a homogenous appearance, sometimes presenting with a "speckled" or a "stippling" perimeter. TEE, as compared to TTE, is a better technique for detecting and describing the extent and precise attachment of PFEs [1], but sometimes direct inspection during surgery finds supplementary lesions, as was the case of our patient. Unfortunately, at the moment, there are no parameters that can be evaluated in order to classify PFEs into low- or high-risk according to their embolization potential. Tumor mobility, as assessed by echocardiogram, did not prove to be a reliable predictor of embolic events [1]. These abnormal structures can be partly responsible for valve insufficiency or hemodynamic conditions similar to stenosis or are simply superimposed on structures whose dysfunction has a distinctive etiology (e.g. degenerative, functional etc.) as was the case of our patient.

Usually, surgical shave-excision is a highly successful therapy $[1,8]$. A recent study reported, for the first time, a recurrence rate of $1.6 \%$ [1], with important implications concerning patient follow-up. Some of the prevailing theories are that PFEs could be wear-and-tear type lesions, occurring where turbulent flow affects the underlying endothelium, or that they can occur after cardiac surgery.

The particularities of the case consisted of the incidental finding of numerous PFEs with several valvular and nonvalvular localizations in a patient with HOCM, for which complex ultrasosnographic examination established an accurate diagnosis. In conclusion, the diagnosis of PFEs can be established using integrated echocardiography modalities (TTE, TEE, 3D echocardiography) and should be considered as a differential diagnosis when observing intracardiac masses in a patient with HOCM. Follow-up remains crucial in these patients due to the risk of recurrence.

\section{References}

1. Tamin SS, Maleszewski JJ, Scott CG, et al. Prognostic and bioepidemiologic implications of papillary fibroelastomas. J Am Coll Cardiol 2015;65:2420-2429.

2. Gowda RM, Khan IA, Nair CK, Mehta NJ, Vasavada BC, Sacchi TJ. Cardiac papillary fibroelastoma: a comprehensive analysis of 725 cases. Am Heart J 2003;146:404-410.

3. Kumar G, Macdonald RJ, Sorajja P, Edwards WD, Ommen SR, Klarich KW. Papillary fibroelastomas in 19 patients with hypertrophic cardiomyopathy undergoing septal myectomy. J Am Soc Echocardiogr 2010;23:595-598.

4. Shub C, Tajik AJ, Seward JB, et al. Cardiac papillary fibroelastomas. Two-dimensional echocardiographic recognition. Mayo Clin Proc 1981;56:629-633.

5. Ong S, Nanda NC, Barold SS. Two-dimensional echocardiographic detection and diagnostic features of left ventricular papillary fibroelastoma. Am Heart J 1982;103:917-918.

6. Pacini D, Farneti PA, Leone O, Galli R. Cardiac papillary fibroelastoma of the mitral valve chordae. Eur J Cardiothorac Surg 1998;13:322-324.

7. De Virgilio C, Dubrow TJ, Robertson JM, et al. Detection of multiple cardiac papillary fibroelastomas using transesophageal echocardiography. Ann Thorac Surg 1989;48:119-121.

8. Ngaage DL, Mullany CJ, Daly RC, et al. Surgical treatment of cardiac papillary fibroelastoma: a single center experience with eighty-eight patients. Ann Thorac Surg 2005;80:1712-1718.

9. Tsukube T, Ataka K, Taniguchi T, Yokoyama M, Hanioka K. Papillary fibroelastoma of the left atrial appendage: echocardiographic findings. Ann Thorac Surg 2000;70:14161417.

10. Sun JP, Asher CR, Yang XS, et al. Clinical and echocardiographic characteristics of papillary fibroelastomas: a retrospective and prospective study in 162 patients. Circulation 2001;103:2687-2693. 